\title{
Two Stage Wavelet based Image Denoising
}

\author{
Glincy Abraham, Neethu Mohan, Sreekala S, Neethu Prasannan, K P Soman \\ Centre for Excellence in Computational Engineering and Networking \\ Amrita Vishwa Vidyapeetham, Coimbatore, Tamil Nadu, India-641112
}

\begin{abstract}
In this paper we are proposing a 2-stage wavelet based denoising technique. First stage of denoising is performed on the approximation coefficient obtained from the level 1 wavelet decomposition [1] of the noisy image and second stage of denoising is applied on the reconstructed image. The second stage denoising has shown a better result on to the reconstructed image. The detail coefficients are newly estimated from the first level denoised approximation coefficients. For denoising, techniques like Total Variation [3], Split Bregman [4] and NL means [5] are used. The quality of results obtained from different denoising techniques has been measured using various objective matrices such as PSNR, MSE on standard test images.
\end{abstract}

\section{General Terms}

Denoising, Wavelet decomposition

\section{Keywords}

Total Variation, Split-Bregman, NL-means, Edge detection

\section{INTRODUCTION}

Denoising is an important preprocessing technique in image processing, which removes the noise while preserving the image quality [8]. In order to retain the edge information and to maintain the quality of the denoised image, wavelet based decomposition [9] was introduced along with conventional image denoising [2]. For estimating the performance of this technique various wavelets and denoising techniques are applied on the standard images.

Nowadays Wavelet transforms has become a powerful computational tool and plays a significant role in image processing. DWT (Discrete Wavelet Transform) decomposes the input image into approximation and detail coefficients. In this paper, we applied the denoising techniques on these approximation coefficients and these denoised coefficients are used for the estimation of new detail coefficients (horizontal, vertical and diagonal). The denoised approximation and estimated detail coefficients are used for the reconstruction of the image. Amount of noise in this reconstructed image will be considerably less. Second stage denoising helps in further filtering of noises in the image and is shown in section 4 . This estimation is done with different wavelets such as 'haar', 'db2', 'bior2.4'.

The techniques used in this paper for denoising include TV denoising [3] Split-Bregman [4] and NL-Means [5]. TV denoising is very effective denoising technique which removes the noise by solving a nonlinear minimization equation. Split Bregman is one of the fastest algorithms for solving through convex optimization. NL-means is another denoising technique based on non-local averaging of pixels. For efficient edge detection Canny [6] method proposed by John F Canny is used, which uses multi-stage algorithm.
Canny uses thresholding with hysteresis which requires two thresholds - high and low.

Section 2 gives an over view of various denoising techniques. Section 3 introduces the proposed method. Section 4 discusses the results for various inputs and its quality measurements.

\section{DENOISING TECHNIQUES}

\subsection{Total Variation}

Total variation based filtering was introduced by Rudin, Osher, and Fatemi [3]. Total variation denoising is applicable in many digital images processing for reducing the noise. The total variation (TV) of a signal measures how much the signal changes between signal values. Total variation of an $\mathrm{N}$ point signal $X(n), 1 \leq n \leq N$ is defined as

$T V(x)=\sum_{n=2}^{N}|x(n)-x(n-1)|$

For an image $u(x, y)$, then TV to be defined as,

$$
\begin{aligned}
& T V(u)=\sum_{i=1}^{M-1} \sum_{j=1}^{N-1}|u(i, j)-u(i+1, j)|+ \\
& |u(i, j)-u(i, j+1)|+\sum_{i=1}^{M-1}|u(i, N)-u(i+1, N)| \\
& +\sum_{j=1}^{M-1}|u(M, j)-u(M, j+1)|
\end{aligned}
$$

The objective function for total variation can be defined as

$$
\begin{aligned}
& u_{t}=\frac{\partial}{\partial x}\left[\frac{u_{x}}{\sqrt{u_{x}^{2}+u_{y}^{2}}}\right]+\frac{\partial}{\partial y}\left[\frac{u_{y}}{\sqrt{u_{x}^{2}+u_{y}^{2}}}\right] \\
& -\lambda\left(u-u_{0}\right)
\end{aligned}
$$

Where $\lambda$ is the regularization parameter, which controls how much smoothing is done. For higher values of $\lambda$ denoised image is very similar to original noisy image. If it is too small value edge information cannot preserve, so we have to choose an optimum value for $\lambda$.

\subsection{Split-Bregman}

Split-Bregman is a suitable technique for solving total variation minimization problem based on partial differential equations. Optimization problems of the following type can be solved by using Split-Bregman technique

$$
\phi: X \rightarrow R H: X \rightarrow R
$$

Constrained function can be defined as 


$$
\min _{u \in X}\|\phi(u)\|_{1}+H(u)
$$

Constrained minimization problem with a subjected to condition can be expressed as,

$$
\min _{u \in X, v \in R}\|v\|_{1}+H(u)+(\lambda / 2)\|d-\phi(u)\|_{2}^{2}
$$

Then the simplified form of Split Bregman can be given as

$$
\begin{aligned}
& \left(u^{k+1}, d^{k+1}\right)=\min _{u \in X, d \in R} J(u, d)-J\left(u^{k}, d^{k}\right)+ \\
& \lambda\left\langle b^{k}, \phi_{u}-\phi_{u}{ }^{k}\right\rangle-\lambda\left\langle b^{k}, d-d^{k}\right\rangle+\lambda / 2\|d-\phi(u)\|_{2}^{2}(6) \\
& \left(u^{k}, d^{k}\right)=\min _{u \in X, d \in R} J(u, d)+(\lambda / 2)\left\|d-\phi(u)-b^{k}\right\|_{2}^{2}+C_{2}(7)
\end{aligned}
$$

Where $C_{2}$ is a constant.

\section{Split-Bregman Algorithm [7]}

$$
\begin{aligned}
& \text { Intialize } k=0 u^{0}=0 b^{0}=0 \\
& \text { while }\left\|u^{k}-u^{k-1}\right\|_{2}^{2}>\text { tol } d o \\
& u^{k+1}=\min _{u} H(u)+\frac{\lambda}{2}\left\|d^{k}-\phi(u)-b^{k}\right\|_{2}^{2} \\
& d^{k+1}=\min _{d}|d|+\frac{\lambda}{2}\left\|d-\phi\left(u^{k+1}\right)-b^{k}\right\|_{2}^{2} \\
& b^{k+1}=b^{k}+\|\left(\phi\left(u^{k+1}\right)-b^{k} \|_{2}^{2}\right. \\
& k=k+1 \\
& \text { end while }
\end{aligned}
$$

\subsection{Non Local Means Algorithms}

It is one of the denoising techniques based on non local averaging of all the pixels in the image. Noise contains both high and low frequency components. Earlier techniques focus on removing the high frequency noise at the cost of high frequency fine details of the original image. These techniques take least care in removing the low frequency noises. Nonlocal means is an algorithm that takes care of the loss of details.

The non local means algorithm considers the extensive amount of self similarity of pixels in an image[10]. Figure 1 [5] explains the self similarity of pixels in an image. Consider the pixel-points $\mathrm{p}, \mathrm{q}_{1}$ and $\mathrm{q}_{2}$ and their respective neighborhood points. The neighborhoods of pixel $\mathrm{p}$ and $\mathrm{q}_{1}$ are similar but that of $\mathrm{q}_{2}$ are not similar, which means most pixels in same columns of $p$ will have similar neighborhood as that of $p$. Pixels with similar neighborhoods can be used to denoised an image. The weighted intensity average of pixels with similar value provides the new denoised value.

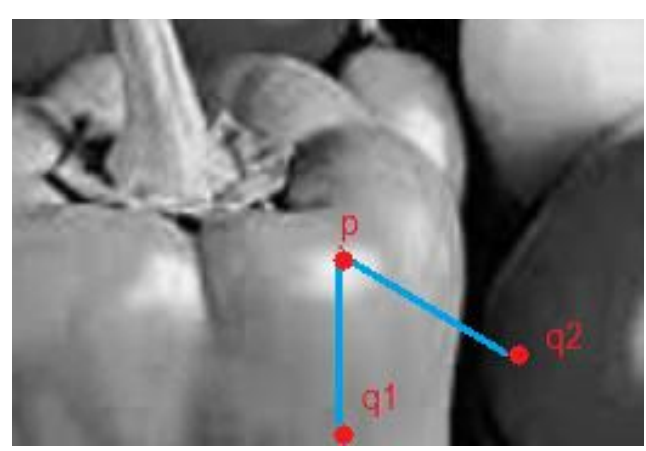

Fig 1. Example of self similarity in an image

The continuous version of NLM can be written as

$u(x)=\frac{\int_{\Omega} w(x, y) u_{o}(y) d y}{\int_{\Omega} w(x, y) d y}$

Where $\quad u_{0}: \Omega \rightarrow R \quad$ is the given noisy image, $u: \Omega \rightarrow R$ is the outcome of the NLM algorithm, function $W$ is defined as

$w(x, y)=e^{\int_{\Omega} G_{a}(z)\left|u_{o}(x+z)-u_{o}(y+z)\right|^{2} d z / h^{2}}$, Where

$G_{a}(z)\left|u_{o}(x+z)-u_{o}(y+z)\right|^{2} d z$ is the distance between patches located at $x, y . h$ is positive constant which acts as scale parameter. $G a$ is Gaussian function with standard deviation $a$.

\section{PROPOSED METHOD}

Two stage wavelet based denoising approach combines different image processing techniques, wavelet based image decomposition, edge detection, for obtaining the denoised image from the noisy image without compromising the quality of the image. Conventional TV denoising requires large number of iterations for removing the Gaussian noise. And large number of iterations will lead to more blurring of the image. In this paper since two stage denoising is employed, less number of iterations happened. At first, the denoised image is decomposed into approximation and detail coefficients using DWT. The output of the Wavelet decomposition is shown in figure 2. The approximation coefficient contains the low frequency component and detail coefficients contain the high frequency component of the input image. One of the denoising techniques was applied with less number of iterations to the approximation coefficient. In order to get the edge information for retaining the information in the detail coefficients, the horizontal, vertical and diagonal differences of this denoised approximation image are estimated and Canny edge detection technique was used for finding the edges. Coefficients corresponding to these edge locations are retained in the detail coefficients and remaining are equated to zero. Denoised approximate coefficient and estimated detailed coefficients are used for wavelet reconstruction. This reconstructed image contains little amount of noise. Second stage denoising is used for removing this noise. Same denoising technique is used in both the stages. Block diagram for this method is shown in figure 3. Outputs obtained in different stages of this technique are shown in figure 4. 


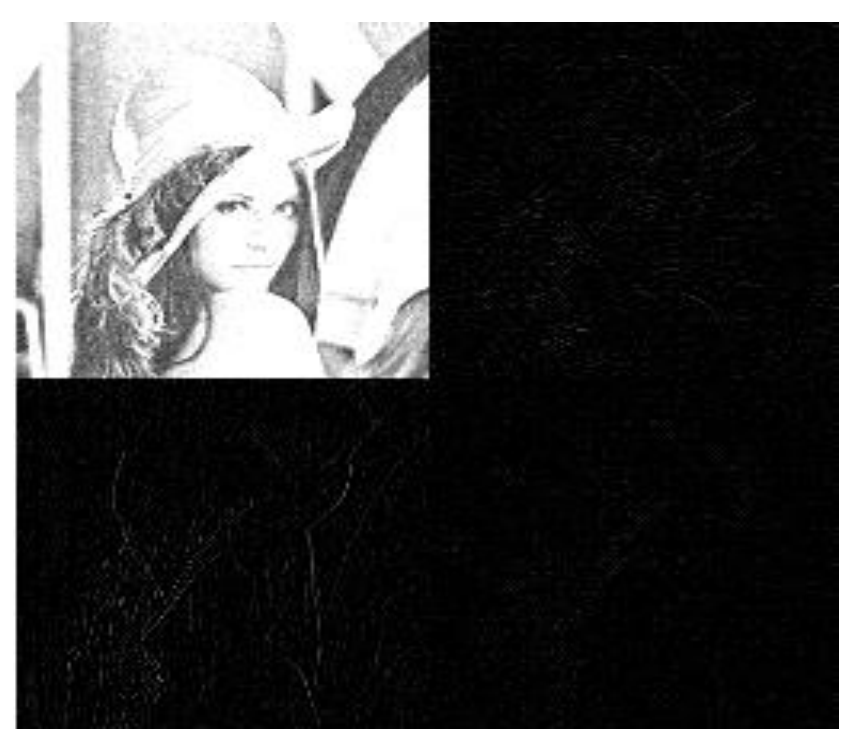

Fig 2: Approximation and detailed coefficient obtained by using DWT.

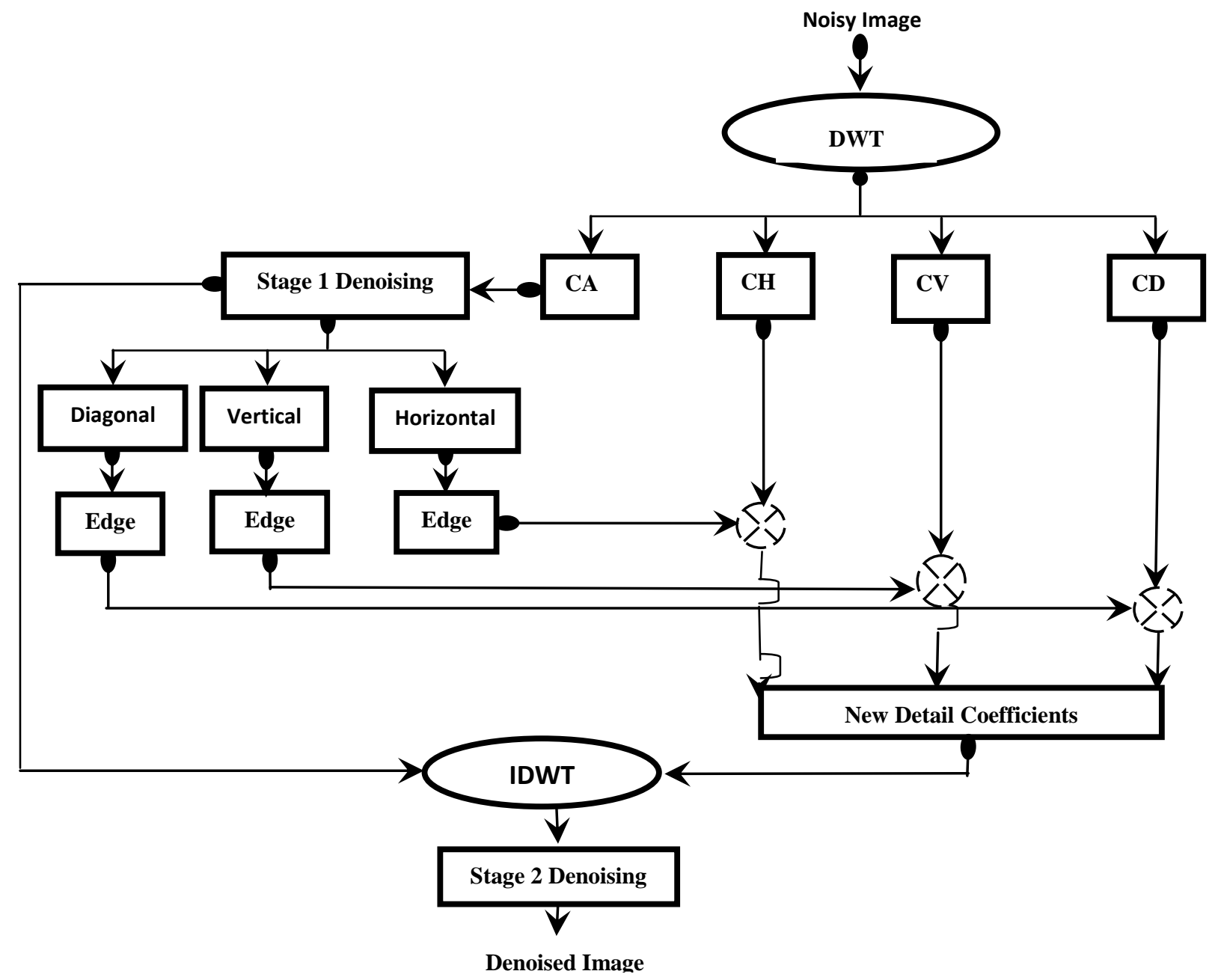

Fig 3: Block diagram of the proposed method 


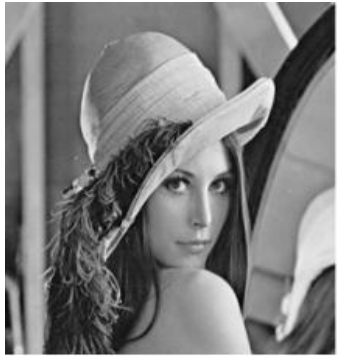

(a)

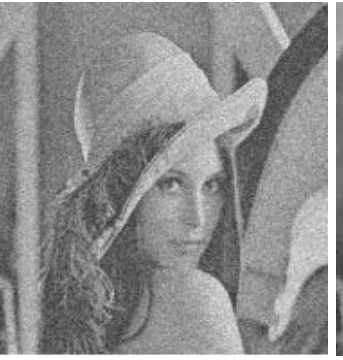

(b)

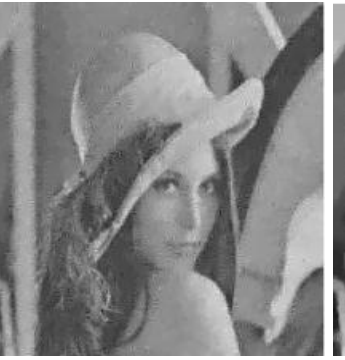

(c)

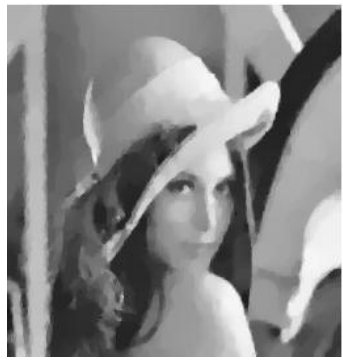

(d)

Fig 4. The performance of the proposed algorithm for lena image with SNR of $25 \mathrm{~dB}$ a)original image b) noisy image c)first stage denoised image d)second stage denoised image. Bregaman denoising and bior2.4 wavelet is used

\section{RESULTS AND DISCUSSION}

For doing experiment Matlab 2009 is been used. Proposed method uses three different wavelets such as 'haar', 'db2', 'bior2.4' and different standard images (clean images) such as 'lena', 'cortex', and 'pepper'. The clean images used are shown in figure. Additive white gaussian noise are added to these clean images with varying SNR (Signal to Noise Ratio) levels such as $10 \mathrm{~dB}, 15 \mathrm{~dB}, 20 \mathrm{~dB}, 25 \mathrm{~dB}, 30 \mathrm{~dB}$ and $35 \mathrm{~dB}$. The result obtained by using Total variation, NL- means and Split Bregman are shown below with the help of figures, tables. The metrics used to evaluate the method are PSNR (PeakSignal-to-Noise ratio) and MSE (Mean Square Error). The SNR level for the figure is $25 \mathrm{~dB}$. The four images in each figure represents original image, noise added image, first stage denoised image and second stage denoised image.

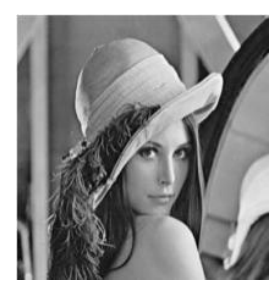

(a)

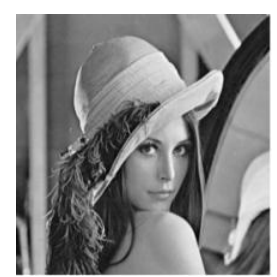

(a)

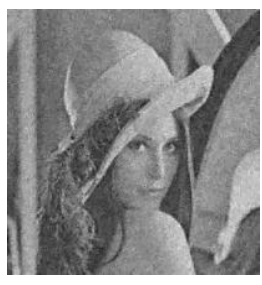

(b)

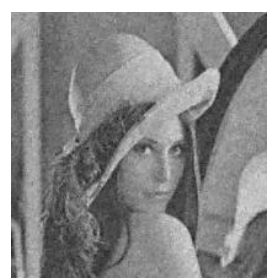

(c)

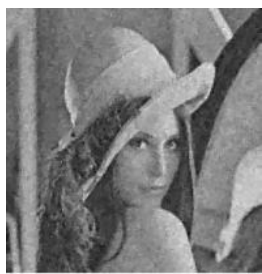

(d)

Fig 5: Result of TV method

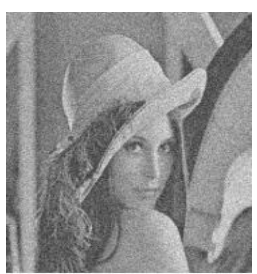

(b)

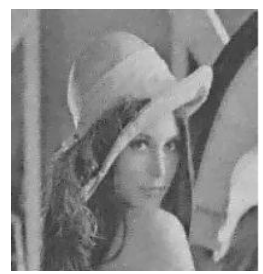

(c)

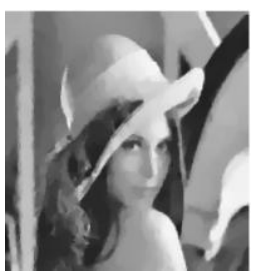

(d)

Fig 6: Result of Split Bregman method

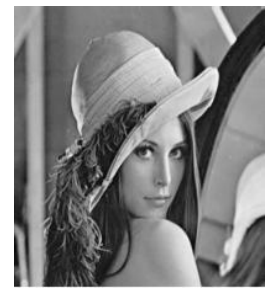

(a)

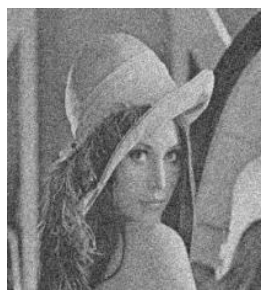

(b)

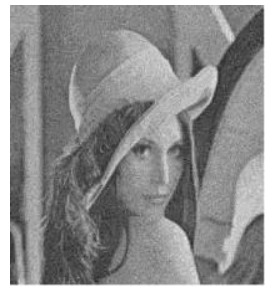

(c)

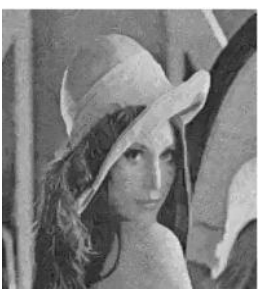

(d)

Fig 7: Result of NLmeans method 


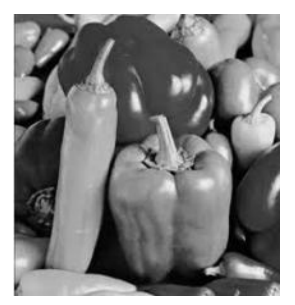

(a)

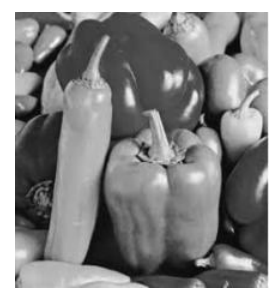

(a)

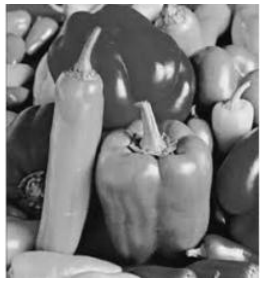

(a)

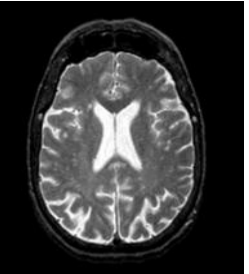

(a)

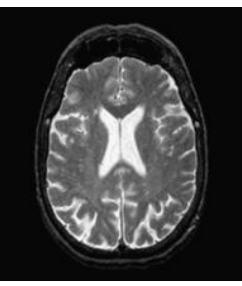

(a)

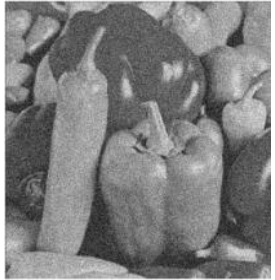

(b)

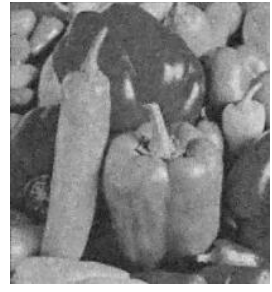

(c)

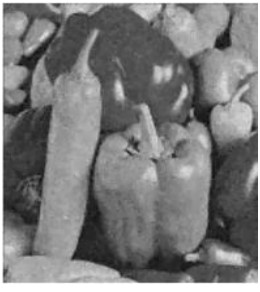

(d)

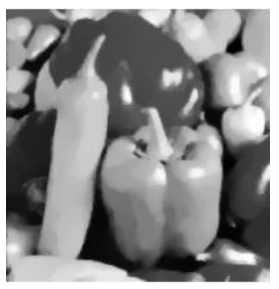

(d)

Fig9: Result of Split Bregman method

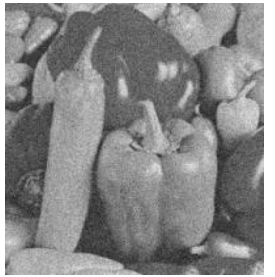

(b)

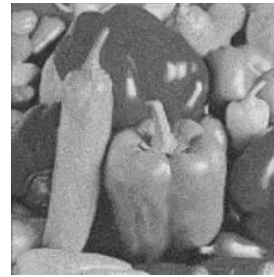

(c)

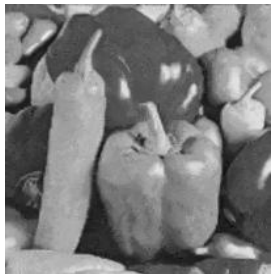

(d)

Fig10: Result of NLmeans method

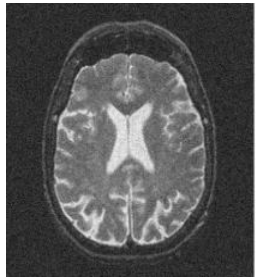

(b)

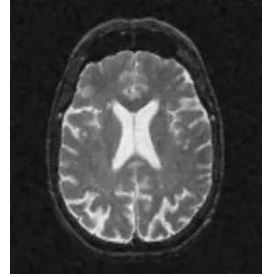

(c)

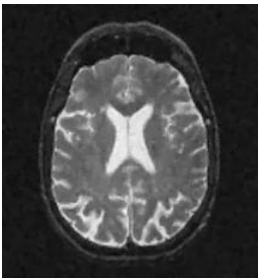

(d)

Fig11: Result of TV method

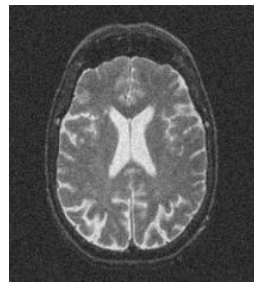

(b)

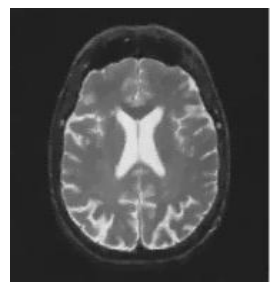

(c)

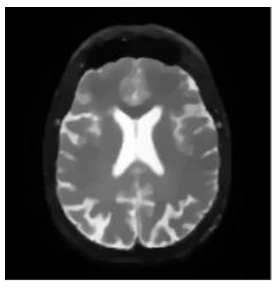

(d)

Fig12: Result of Split Bregman method 


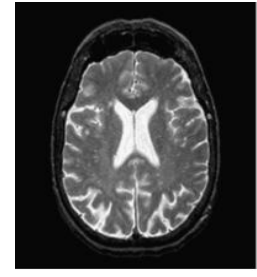

(a)

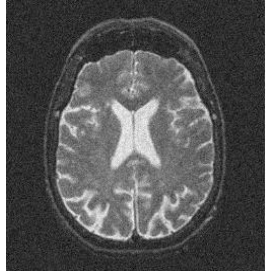

(b)

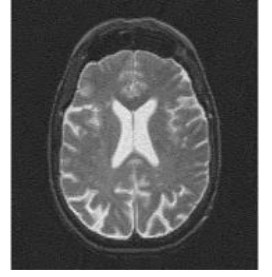

(c)

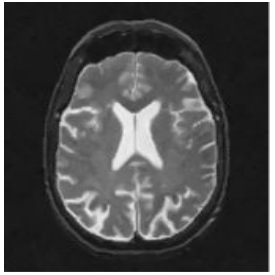

(d)

Fig13: Result of NLmeans method

Outputs obtained by applying three denoising techniques in different images with SNR 25db and Bior2.4 wavelet. a) Original image b)Noisy image c)First Level Denoised Image d) Second Level Denoised Image

Table1: PSNR and MSE values for various SNR, denoising technique and wavelets applied on different images

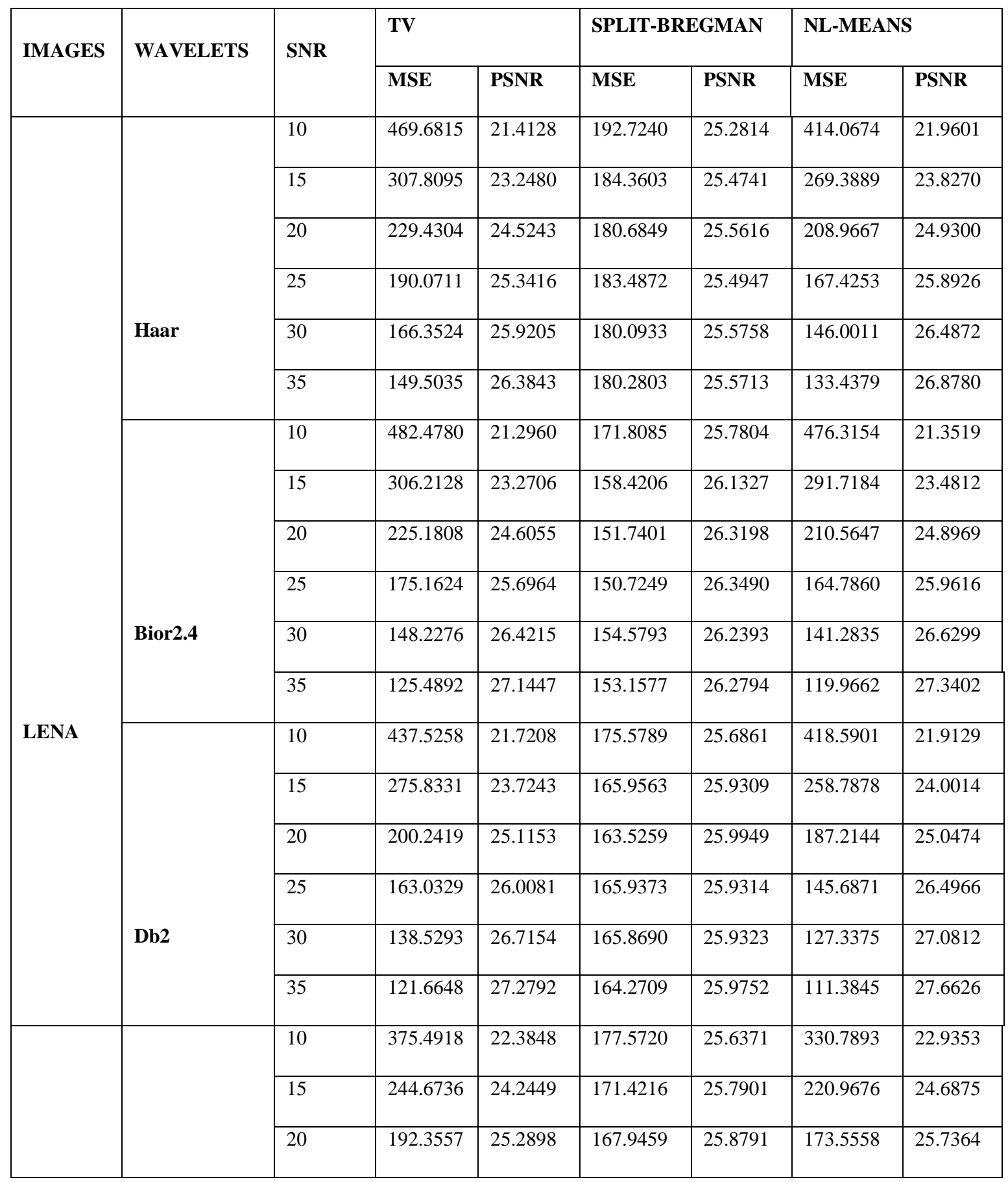




\begin{tabular}{|c|c|c|c|c|c|c|c|c|}
\hline \multirow{15}{*}{ PEPPER } & \multirow[t]{3}{*}{ Haar } & 25 & 157.3129 & 26.1632 & 166.3207 & 25.9213 & 146.7435 & 26.4652 \\
\hline & & 30 & 140.4432 & 26.6558 & 167.3562 & 25.8944 & 130.7190 & 26.9674 \\
\hline & & 35 & 128.8912 & 27.0286 & 165.4439 & 25.9443 & 121.6589 & 27.2794 \\
\hline & \multirow{6}{*}{ Bior2.4 } & 10 & 357.5616 & 22.5973 & 146.2815 & 26.4789 & 361.8132 & 22.5460 \\
\hline & & 15 & 220.0797 & 24.7050 & 137.0415 & 26.7623 & 219.8704 & 24.7091 \\
\hline & & 20 & 161.1183 & 26.0594 & 129.8188 & 26.9974 & 156.9398 & 26.1735 \\
\hline & & 25 & 126.8048 & 27.0994 & 131.9146 & 26.9279 & 125.2639 & 27.1525 \\
\hline & & 30 & 106.5310 & 27.8560 & 130.1494 & 26.9864 & 109.3381 & 27.7431 \\
\hline & & 35 & 94.4527 & 28.3787 & 130.5413 & 26.9733 & 96.3602 & 28.2918 \\
\hline & \multirow{6}{*}{ Db2 } & 10 & 329.8899 & 22.9471 & 153.3561 & 26.2738 & 319.2815 & 23.0891 \\
\hline & & 15 & 205.4821 & 25.0031 & 148.6414 & 26.4094 & 194.5471 & 25.2406 \\
\hline & & 20 & 151.1988 & 26.3353 & 142.5305 & 26.5917 & 143.5987 & 26.5593 \\
\hline & & 25 & 122.8566 & 27.2368 & 141.7675 & 26.6150 & 119.6524 & 27.3516 \\
\hline & & 30 & 105.1201 & 27.9139 & 143.8016 & 26.5532 & 103.5709 & 27.9784 \\
\hline & & 35 & 93.5434 & 28.4207 & 141.4719 & 26.6241 & 92.9523 & 28.4482 \\
\hline \multirow{16}{*}{ CORTEX } & \multirow{6}{*}{ Haar } & 10 & 173.4387 & 25.7393 & 192.8844 & 25.2778 & 171.4136 & 25.7904 \\
\hline & & 15 & 133.0572 & 26.8904 & 192.7704 & 25.2804 & 133.3394 & 26.8812 \\
\hline & & 20 & 117.0054 & 27.4487 & 194.0108 & 25.2525 & 122.7949 & 27.2390 \\
\hline & & 25 & 106.4295 & 27.8602 & 191.7412 & 25.3036 & 114.7209 & 27.5344 \\
\hline & & 30 & 101.8293 & 28.0521 & 192.4496 & 25.2876 & 111.4165 & 27.6613 \\
\hline & & 35 & 96.9273 & 28.2663 & 191.6156 & 25.3065 & 107.9106 & 27.8002 \\
\hline & \multirow{6}{*}{ Bior2.4 } & 10 & 147.0779 & 26.4553 & 151.4136 & 26.3292 & 157.2083 & 26.1660 \\
\hline & & 15 & 99.9823 & 28.1316 & 151.6972 & 26.3210 & 107.4039 & 27.8206 \\
\hline & & 20 & 80.6164 & 29.0965 & 151.9697 & 26.3132 & 91.4771 & 28.5177 \\
\hline & & 25 & 69.7430 & 29.6958 & 152.6894 & 26.2927 & 82.1508 & 28.9847 \\
\hline & & 30 & 64.9670 & 30.0039 & 152.8228 & 26.2889 & 77.4640 & 29.2398 \\
\hline & & 35 & 58.6304 & 30.4496 & 152.46 & 26.2992 & 72.8118 & 29.5088 \\
\hline & \multirow[b]{4}{*}{ Db2 } & 10 & 137.1325 & 26.7594 & 169.1666 & 25.8477 & 143.4448 & 26.5640 \\
\hline & & 15 & 95.6656 & 28.3232 & 167.9859 & 25.8781 & 101.1889 & 28.0795 \\
\hline & & 20 & 80.5492 & 29.0702 & 170.2718 & 25.8194 & 89.9937 & 28.5887 \\
\hline & & 25 & 73.5569 & 29.4646 & 166.6787 & 25.9120 & 83.3093 & 28.9239 \\
\hline
\end{tabular}




\begin{tabular}{|l|l|l|l|l|l|l|l|l|}
\hline & 30 & 67.1340 & 29.8614 & 169.1139 & 25.8490 & 79.2112 & 29.1429 \\
\cline { 3 - 8 } & & 35 & 64.7134 & 30.0209 & 168.5356 & 25.8639 & 77.3656 & 29.2453 \\
\hline
\end{tabular}

\section{CONCLUSION}

In this paper, a two stage wavelet based denoising method is presented. Experiments are conducted on 3 different images with different SNR values and the results for various denoising techniques are compared. The proposed method gives good results for different SNR values as seen in the table. Db2 and Bior2.4 wavelet gives better results compared with Haar. Here in denoising, only the detailed coefficients obtained from the dwt are retained, keeping the rest of the coefficients as zero. In future, denoised versions of these coefficients could also be utilized to provide better result.

\section{REFERENCES}

[1] Stephane G Mallat "A theory for multiresolution signal decomposition:The wavelet representation"IEEE Transactions On Pattern Analysis And Machine Intelligence, Vol 11, No. 7. July 1989

[2] Nilamani Bhoi, Dr. Sukadev Meher" Total Variation based Wavelet Domain Filter for Image Denoising" First International Conference on Emerging Trends in Engineering and Technology

[3] L. Rudin, S. Osher, and E. Fatemi. Nonlinear total variation based noise removal algorithms. PhysicaD, 60:259-268, 1992
[4] Jacqueline Bush "Bregman Algorithms" Senior Thesis. University of California, Santa Barbara, June 10, 2011

[5] A.Buades, B.Coll, and J Morel "A non-local algorithm for image denoising", IEEE International Conference on Computer vision and Pattern Recognition,2005

[6] John Canny, member, IEEE, "A Computational Approach to Edge Detection", IEEE Transactions on Pattern Analysis and Machine Intelligence, VOL. PAMI8, NO. 6, November 1986

[7] Bregman Algorithms Author: JacquelineBush Supervisor Dr.CarlosGarc' 1a-Cervera June 10, 2011

[8] R C.Gonzalez and R.E Woods,Digital Image Processing, Addison Wesley Longman Inc.,2000.

[9] Kossi Edoh and John Paul Roop "A Fast Wavelet Multilevel Approach to Total Variation Image Denoising”, International Journal of Signal Processing, Image Processing and Pattern Recognition Vol. 2, No.3,September 2009.

[10] Dr. K.P.Soman, R.Ramanathan "Chapter 25, Digital Signal and Image Processing- The Sparse Way" 2012 by Isa Publishers. 